\title{
Article
}

\section{Biochemical Studies of the Lagunamides, Potent Cytotoxic Cyclic Depsipeptides from the Marine Cyanobacterium Lyngbya majuscula}

\author{
Ashootosh Tripathi ${ }^{1}$, Wanru Fang ${ }^{2}$, David Tai Leong ${ }^{2, *}$ and Lik Tong Tan ${ }^{1, *}$ \\ 1 Natural Sciences and Science Education, National Institute of Education, Nanyang Technological \\ University, 1 Nanyang Walk, 637616, Singapore; E-Mail: ashootosh.tripathi@gmail.com \\ 2 Department of Chemical and Biomolecular Engineering, National University of Singapore, \\ 4 Engineering Drive, 117576, Singapore; E-Mail: chefangw@nus.edu.sg \\ * Authors to whom correspondence should be addressed; E-Mails: cheltwd@nus.edu.sg (D.T.L.); \\ liktong.tan@nie.edu.sg (L.T.T.); Tel.: +65-6516-7262 (D.T.L.); +65-6790-3842 (L.T.T.); \\ Fax: +65-6779-1936 (D.T.L.); +65-6896-9414 (L.T.T.).
}

Received: 17 April 2012; in revised form: 9 May 2012 / Accepted: 10 May 2012 /

Published: 23 May 2012

\begin{abstract}
Lagunamides A (1) and B (2) are potent cytotoxic cyclic depsipeptides isolated from the filamentous marine cyanobacterium, Lyngbya majuscula, from Pulau Hantu, Singapore. These compounds are structurally related to the aurilide-class of molecules, which have been reported to possess exquisite antiproliferative activities against cancer cells. The present study presents preliminary findings on the selectivity of lagunamides against various cancer cell lines as well as their mechanism of action by studying their effects on programmed cell death or apoptosis. Lagunamide A exhibited a selective growth inhibitory activity against a panel of cancer cell lines, including P388, A549, PC3, HCT8, and SK-OV3 cells, with $\mathrm{IC}_{50}$ values ranging from $1.6 \mathrm{nM}$ to $6.4 \mathrm{nM}$. Morphological studies showed blebbing at the surface of cancer cells as well as cell shrinkage accompanied by loss of contact with the substratum and neighboring cells. Biochemical studies using HCT8 and MCF7 cancer cells suggested that the cytotoxic effect of $\mathbf{1}$ and $\mathbf{2}$ might act via induction of mitochondrial mediated apoptosis. Data presented in this study warrants further investigation on the mode of action and underscores the importance of the lagunamides as potential anticancer agents.
\end{abstract}

Keywords: marine cyanobacterium; cyclic depsipeptides; lagunamides; apoptosis; cytotoxicity 


\section{Introduction}

Benthic filamentous marine cyanobacteria are a source of novel bioactive compounds. To date more than 400 nitrogen-containing molecules have been reported in the literature, belonging mostly to the polyketide-polypeptide structural class of natural products [1]. The importance of this structural class is attested by a number of natural products, including bleomycin, rapamycin, and FK506, currently being used as pharmaceuticals [2]. A high proportion of marine cyanobacterial compounds, such as dolastatin 10, apratoxin A, and largazole, have shown to possess exquisite potency and have subsequently been considered for further development as therapeutic agents, especially in the area of cancer research. The high potencies of these compounds are due to their specific interference with cellular targets, including microtubules, actin filaments, and histone deacetylase [3]. To date, a dolastatin 10-analogue, auristatin E, has been formulated as an antibody drug conjugate, brentuximab vedotin, and approved for the treatment of Hodgkin lymphoma and anaplastic large cell lymphoma [4]. In addition, at least three antibody drug conjugates based on synthetic analogues of marine cyanobacterial compounds are currently in the clinical pipeline as anticancer agents [5].

Recent research by Tan and co-workers revealed the chemical richness of a persistent strain of the marine cyanobacterium, Lyngbya majuscula, collected from Pulau Hantu, Singapore. At least a dozen compounds, both new (e.g. hantupeptins and besarhanamides) and known have been reported from this strain [6-8]. A recollection of this particular cyanobacterial strain in 2007 yielded two cyclodepsipeptides, lagunamides $\mathrm{A}$ and $\mathrm{B}$, with significant cytotoxicity having $\mathrm{IC}_{50}$ values of 6.4 and $20.5 \mathrm{nM}$, respectively, when tested against the P388 murine leukemia cell line [9]. These compounds belong to the polyketide-polypeptide natural products and are structurally related to the aurilide-class of molecules. In addition, significant antimalarial activities were also reported for the lagunamides, a first for this class of molecules [9]. Due to the exquisite potency of the lagunamides as well as being available in sufficient quantities, they were selected for further biological testing, in particular, for their effects on apoptosis or cell death.

The apoptotic pathway has become a major target for developing anticancer drugs due to the conserved pathway of programmed cell death [10]. During embryonic development, cell death is essential for successful organogenesis and the formation of complex multicellular tissues [11]. Apoptosis also operates in adult organisms to maintain normal cellular homeostasis, and resistance to apoptosis is a hallmark of cancer, often contributing to chemoresistance. Several key apoptotic pathways are altered in cancer, including the "loss of function" mutations in the p53 tumor suppressor gene, as well as the overexpression of anti-apoptotic proteins such as the mitochondrial anti-oncogenes Bcl-2 and Bcl- $\mathrm{X}_{\mathrm{L}}[12]$. Herein are preliminary biological data conducted on the lagunamides to profile their cytotoxic activities against a panel of cancer cell lines as well as their effect on the apoptotic pathway based on a biochemical method.

\section{Results and Discussion}

\subsection{Structures of Lagunamides and the Aurilide-Class of Molecules}

Lagunamides A (1) and B (2) (Chart 1) are cyclic depsipeptides recently isolated from the marine cyanobacterium, Lyngbya majuscula, obtained from the shallow lagoon at Pulau Hantu, Singapore [9]. 
The planar structures were established primarily by 1D and 2D NMR data as well as MS spectral data. These compounds belong to the mixed polyketide-polypeptide structural class, consisting of five amino acids (Ala, $N \mathrm{Me}$-Phe, $\mathrm{NMe}-\mathrm{Gly}$, Ile, and $\mathrm{NMe}$-Ala), one-hydroxy acid (2-hydroxy-isoleucic acid) and a unique extended polyketide moiety. The lagunamides are structurally related to the aurilide class of molecules, which include aurilides B (4) and C (5) and kulokekahilide-2 (6) (Chart 1) [13-15]. Aurilide (3), the first member of this class of molecules, was originally reported from the sea hare, Dolabella auricularia [16]. However, it has been speculated that marine cyanobacteria are the true source of the compound and the presence of aurilide in the animal is due to sequestration via the invertebrate's diet. There is growing interest in the development of these molecules as potential anticancer agents as attested by a number of synthetic efforts geared towards their total synthesis as well as generation of analogues for structural activity relationship (SAR) studies [15,17-21].

Chart 1. Chemical structures of lagunamides (1 and 2), aurilides (3-5), and kulokekahilide-2 (6).

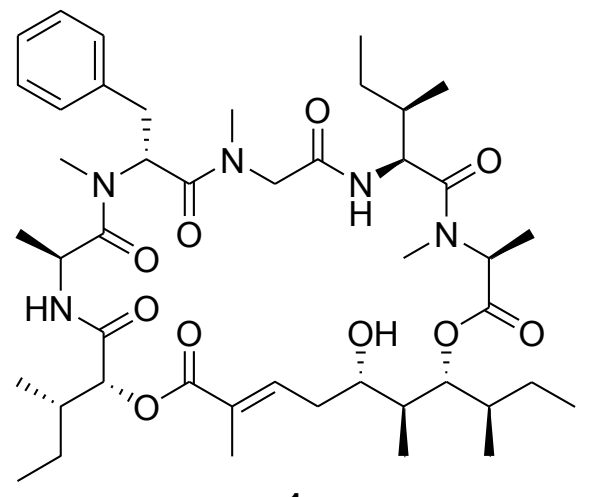

1

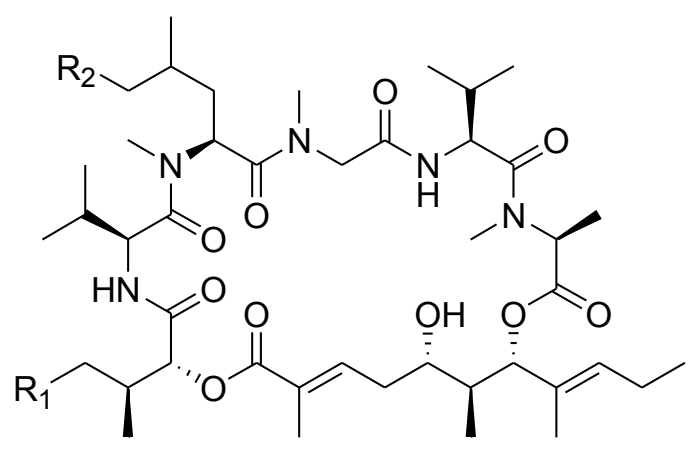

$3 \mathrm{R}_{1}=\mathrm{CH}_{3}, \mathrm{R}_{2}=\mathrm{H}$ (D-MeLeu)

$4 \mathrm{R}_{1}=\mathrm{CH}_{3}, \mathrm{R}_{2}=\mathrm{CH}_{3}$ (allo-L-Melle)

$5 \quad \mathrm{R}_{1}=\mathrm{H}, \mathrm{R}_{2}=\mathrm{CH}_{3}$ (allo-L-Melle)

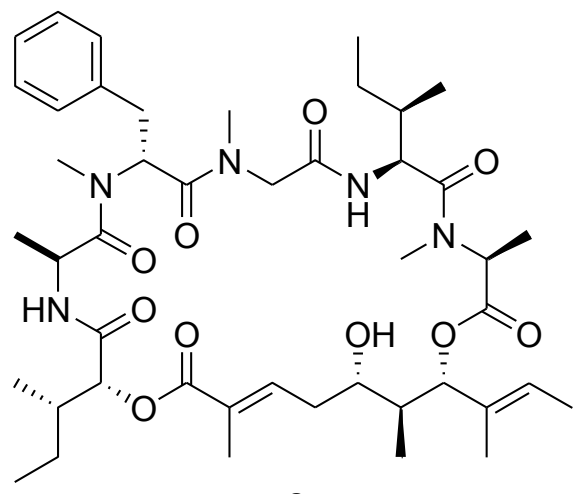

2

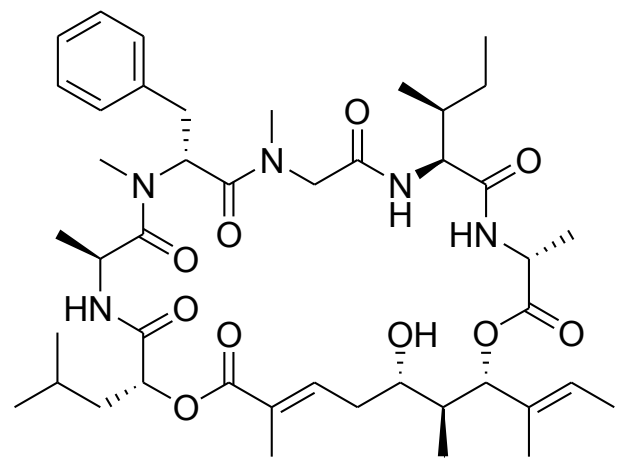

6

\subsection{Evaluation of Lagunamide A against BJ and BJ shp53 Cell Lines}

Lagunamide A (1) was originally reported to induce cytotoxicity in the P388 murine leukemia cell line with an $\mathrm{IC}_{50}$ of $6.4 \mathrm{nM}$ [9]. Subsequently, it was decided to first test 1 against human foreskin fibroblast cells (BJ) and p53 tumor suppressor oncogene knocked down fibroblast cells (BJ shp53). The rationale was to elucidate if the cytotoxic nature of $\mathbf{1}$ was specific towards normal cell types and also to determine if p53 expression could account for any alteration in activities. Compound 1 displayed $\mathrm{IC}_{50}$ values of 20.2 and $58.8 \mathrm{nM}$ when tested against BJ and BJ shp53 cells, respectively 
(Table 1). The differential $\mathrm{IC}_{50}$ values observed in the two cell lines suggested that the $\mathrm{p} 53$ gene may play a role in the sensitivity of BJ cells to $\mathbf{1}$, as disabling p53 function in BJ shp53 reduced the sensitivity by almost 3-fold. Furthermore, time-based experiments carried out on both cell types showed that BJ shp53 (Figures 1 and 2) responded more sensitively when treated at $400 \mathrm{nM}$ over a short span of time of 3 days. However, at other concentrations, 1 caused a significant reduction in proliferation of both BJ and BJ shp53 cells.

Table 1. $\mathrm{IC}_{50}$ values (nM) of lagunamides A (1) and B (2), aurilide (3), aurilides B (4) and $\mathrm{C}(\mathbf{5})$, and kulokekahilide-2 (6) against various cell lines.

\begin{tabular}{|c|c|c|c|c|c|c|c|}
\hline Cell Lines & Cell Types & 1 & 2 & $3^{a}$ & $4^{b}$ & $5^{b}$ & $6^{c}$ \\
\hline $\mathrm{BJ}$ & Foreskin fibroblast & 20.2 & & & & & \\
\hline BJ shp53 & p53 knocked down fibroblast & 58.8 & & & & & \\
\hline P388 & Murine leukemia & 6.4 & 20.5 & & & & 4.2 \\
\hline A549 & Lung adenocarcinoma epithelial & 2.9 & & & & & \\
\hline NCI-H460 & Lung large-cell carcinoma & & & & 10 & 50 & \\
\hline PC3 & Prostate carcinoma & 2.5 & & & & & \\
\hline HCT8 & Ileocecal colorectal adenocarcinoma & 1.6 & 5.2 & & & & \\
\hline SK-OV3 & Ovarian carcinoma & 3.8 & & & & & 7.5 \\
\hline HeLaS3 & Human epithelial carcinoma & & & 11 & & & \\
\hline MDA-MB-435 & Human breast carcinoma & & & & & & 14.6 \\
\hline A10 & Vascular smooth muscle & & & & & & 59.1 \\
\hline Neuro-2a & Mouse neuroblastoma & & & & 40 & 130 & \\
\hline
\end{tabular}

The p53 suppresser oncogene is known to guard against DNA damage, intracellular stresses, and to modulate proliferative signals, thus preventing cells from turning malignant by inducing either cell arrest or apoptosis [22]. Furthermore, there are several pro-apoptotic transcriptional targets of p53, such as BAX and BH-3 proteins, and NOXA and PUMA, which promote cytochrome $c$ release from the mitochondrion [23]. The activity of 1 on fibroblast cells indicates that it might act by stressing the mitochondrion through p53 up-regulation leading to the initiation of the intrinsic apoptotic pathway.

There are generally two pathways that trigger apoptosis: the extrinsic and intrinsic apoptotic pathways [24]. The extrinsic pathway, otherwise known as the "death receptor pathway", is characterized by the activation of death receptors on the cell surface, leading to the initiation of the caspase cascade via caspase 8 . In contrast, the intrinsic pathway involves the permeabilization of mitochondria and release of cytochrome $c$ from mitochondria into the cytoplasm, leading to the activation of the caspase cascade through caspase 9. Whichever pathway is taken, both lead to the activation of various caspase-enzymes responsible for the demise of the cell. The majority of apoptosis in vertebrate systems proceeds through the mitochondrial pathway [25]. 
Figure 1. Cytotoxic effects of lagunamide A (1) against BJ cells over 7 days.

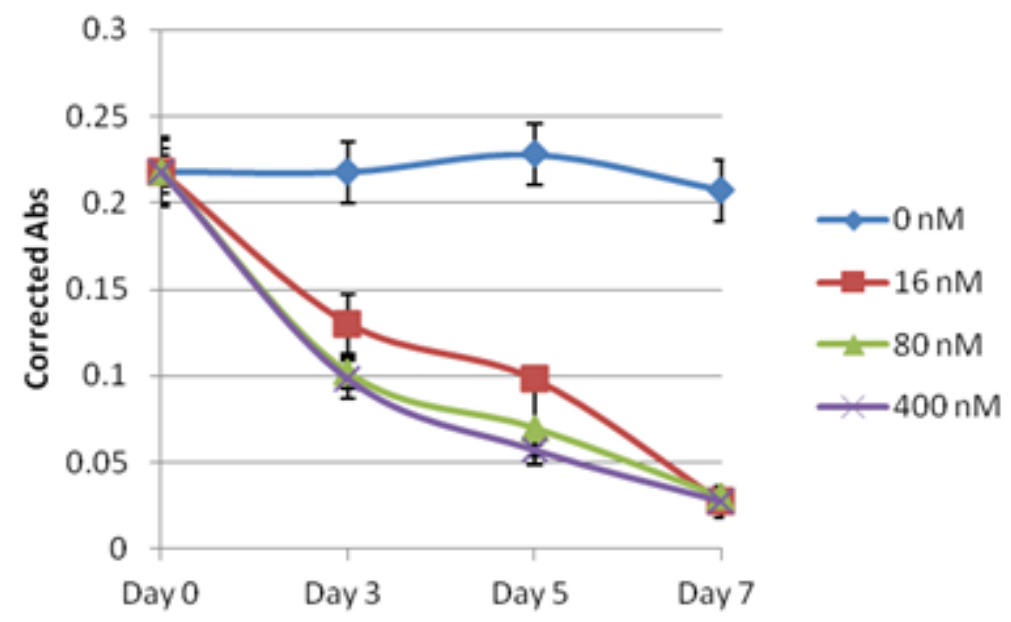

Figure 2. Cytotoxic effects of lagunamide A (1) against BJ shp53 cells over 7 days.

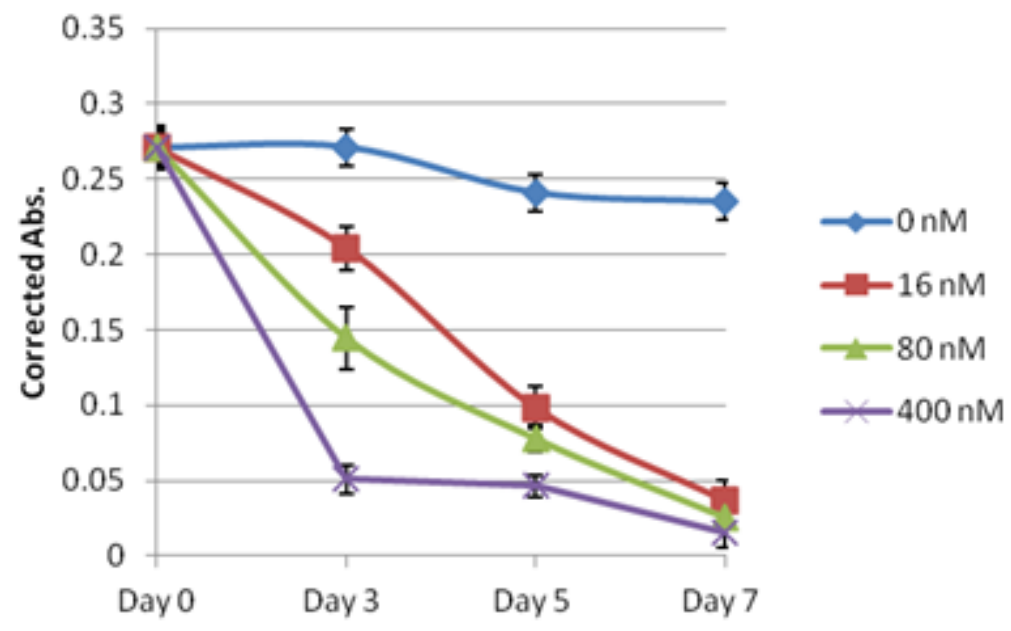

\subsection{Evaluation of Lagunamide A against a Panel of Cancer Cell Lines}

Lagunamide A (1) was next tested against a panel of cancer cell lines, including P388, A549, PC3, HCT8, and SK-OV3 (Table 1). The cytotoxicity data indicated that these cancer cells were more susceptible to $\mathbf{1}$ by approximately four to ten-fold compared to the BJ (Table 1). Due to limited amounts of lagunamide B (2), it was tested only in two cell lines, P388 and HCT8, which showed $\mathrm{IC}_{50}$ values of 20.5 and $5.2 \mathrm{nM}$, respectively. Comparing the two lagunamides, 2 showed reduced cytotoxicity compared to $\mathbf{1}$. Since the only structural difference between $\mathbf{1}$ and $\mathbf{2}$ is presence of the olefinic group in the latter molecule, the enhanced cytotoxicity of compound $\mathbf{1}$ could be due to the non specific toxicity arising from the conjugate addition of cellular nucleophiles by 2 [26]. In addition, the human A549 lung adenocarcinoma epithelial cells used in the assay were reported not to undergo Fas-mediated apoptosis [27]. However, it was observed in this study that A549 cells were sensitive to the cytotoxic effect of $\mathbf{1}$, with an $\mathrm{IC}_{50}$ value of $2.9 \mathrm{nM}$, suggesting that $\mathbf{1}$ might not induce apoptosis through the extrinsic pathway for killing cancer cells.

Selective cytotoxicity towards other cell lines was also observed for other members of the aurilide class of molecules (Table 1). For instance, the $\mathrm{IC}_{50}$ values of kulokekahilide-2 against several cell 
lines, including P388, SK-OV-3, MDA-MB-435, and A-10, ranged from 4.2 to $59.1 \mathrm{nM}$ [13]. When evaluated in the NCI 60 cell lines, aurilide and aurilide B were selective against renal, and prostate cancer cell lines [14,17]. Furthermore, both aurilide and aurilides $\mathrm{B}$ and $\mathrm{C}$ showed significant in vivo antitumor activities based on NCI's hollow fiber assay [14,17]. Given the profile of potent cytotoxicity to cancer cells, the lagunamides join the growing list of aurilide-class molecules as potential leads in cancer treatment.

Morphological observations revealed that the loss of cell proliferation in all cancer cells was associated with cell shrinkage, accompanied by the loss of contact with the substratum and neighboring cells. The detached cells appeared to be spherical with blebbing morphology when observed under high magnifications (Figure 3). Such morphological observations are the hallmark indicators of programmed cell death/apoptosis [28].

Figure 3. Microscopic $(\times 200)$ images of $(\mathbf{A})$ untreated HCT 8 cells and $(\mathbf{B})$ HCT 8 cells treated with lagunamide A (1).

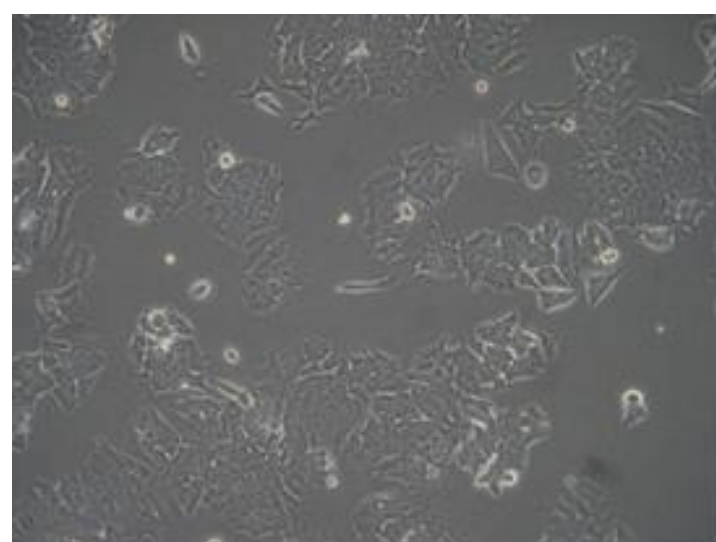

A

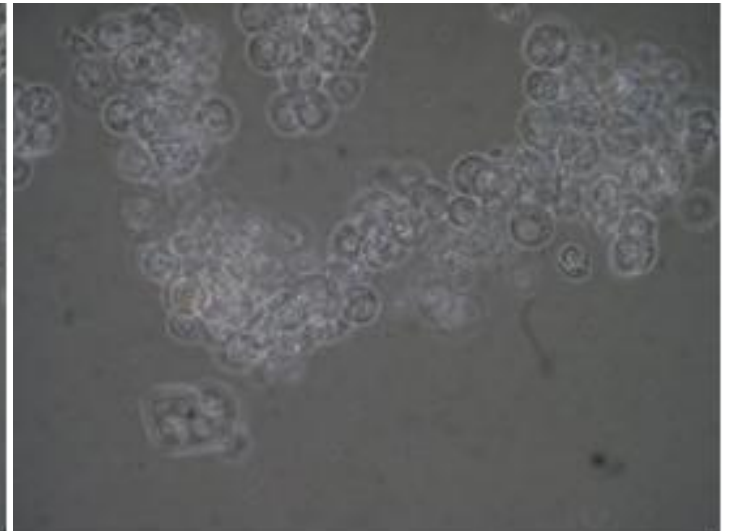

B

\subsection{Biochemical Studies of Lagunamide A Using HCT8 and MCF7 Cell Lines}

Results thus far were suggestive of the intrinsic apoptotic pathway as the probable mode of action associated with lagunamide A (1). An in-depth biochemical analysis was therefore initiated to determine if lagunamides A and B trigger a caspase-mediated apoptosis. As such, Western blot analyses of 1 - or 2 -treated HCT 8 cell lysates with caspase 9, caspase 3 and Bcl- $\mathrm{X}_{\mathrm{L}}$ antibodies were performed (Figure 4). These experiments revealed that caspase 9 was cleaved and activated, suggesting activation of mitochondrial-mediated apoptosis through the release of cytochrome $c$. In addition, expression level of $\mathrm{Bcl}-\mathrm{X}_{\mathrm{L}}$ (anti-apoptosis) remains fairly constant, suggesting no upregulation of the anti-apoptotic response. 1-treated HCT 8 cells demonstrated the highest level of cleaved (active fragment) caspase 9 (Figure 4), indicating the involvement of cytochrome $c$ as well as the nucleotide dATP/ATP which auto-catalytically activates procaspase 9 , as shown by the cleaved caspase 9 band (Figure 4). This, in turn, can proceed to activate downstream executioner caspases, such as caspases-3 and -7 . 
Figure 4. Western blot analysis of lysates from DMSO (-ve ctrl), lagunamide A ( $\mathrm{Lg} \mathrm{A}$ ), lagunamide B (Lg B), and taxol (Txl)-treated HCT8 cells with pro-caspase 9, caspase 9, pro-caspase and $\mathrm{Bcl}-\mathrm{X}_{\mathrm{L}}$ antibodies. The anti-actin antibody was used to ensure equal amounts of protein were loaded.

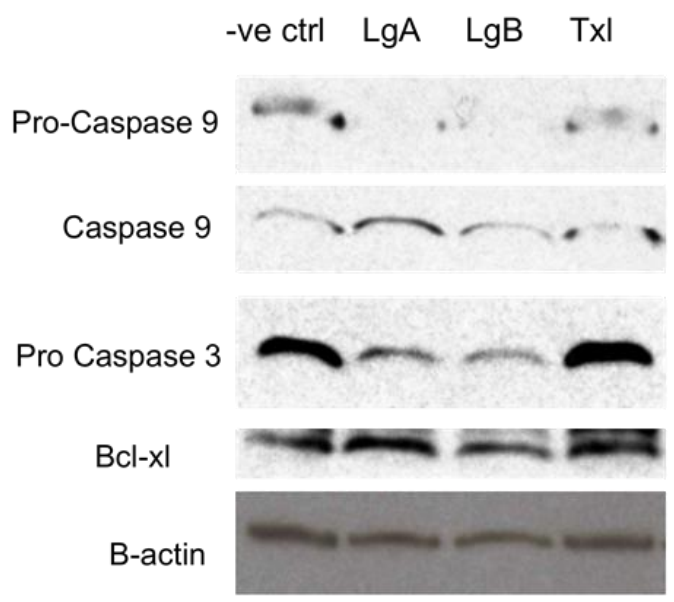

Subsequently, the level of pro-caspase 3 was observed to be lower for cells exposed to 1 and 2 when compared to both positive (taxol-treated) and negative control (DMSO-treated) cell lysates. This suggested the plausible downstream processing of the apoptotic cascade. Cleaved caspase 3, however, was not detected in the experiment. Interestingly, cells treated with 1 also expressed marginally higher levels of Bcl- $\mathrm{X}_{\mathrm{L}}$ (a pro-survival factor) compared to $\mathbf{2}$.

The biochemical study was also extended to MCF7 breast cancer cells treated with $\mathbf{1}$ and 2 (Figure 5). These experiments revealed that 1-treated MCF7 cells had the lowest level of caspase 9 compared to cancer cells treated with taxol and DMSO (negative control). The endogenous levels of pro-caspase 3 were undetected in MCF7 cells, suggesting that it had undergone autocatalysis to initiate a further downstream cascade leading to the intrinsic apoptotic pathway. Moreover, unlike in treated HCT8 cells, Bcl- $\mathrm{X}_{\mathrm{L}}$ was shown to be present at very low levels for all treatments (Figure 5).

Figure 5. Western blot analysis of lysates from DMSO (-ve ctrl), lagunamide A ( $\mathrm{Lg} \mathrm{A}$ ), lagunamide B ( $\operatorname{Lg}$ B), and taxol (Txl)-treated MCF7 cells with pro-caspase 9, caspase 9, pro-caspase 3 and $\mathrm{Bcl}-\mathrm{X}_{\mathrm{L}}$ antibodies. The anti-actin antibody was used to ensure equal amounts of protein were loaded.

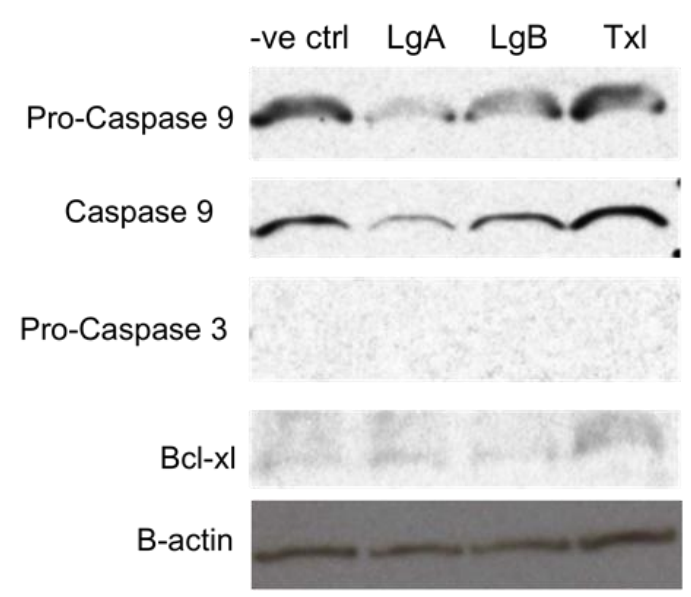


These series of biochemical studies suggested that the cytotoxicity of the lagunamides could be due to either passive diffusion or active transported into the cells, triggering mitochondrial-mediated apoptosis. Recently, a report by Sato and co-workers showed that aurilide induces apoptosis via the mitochondrial-mediated pathway [29]. In addition, the study showed aurilide to selectively target prohibitin 1 (PHB 1) localized within the mitochondria. One of the functions of prohibitin is in the maintenance of mitochondrial function as well as protection from senescence [30]. The inhibition of PHB 1 by aurilide led to the induction of the mitochondrial fragmentation through enhanced processing of the mitochondrial fusion protein optic atrophy 1 (OPA1). This resulted in the loss of membrane potential and the induction of apoptotic cell death [29]. Interestingly, they did not report any downregulation/upregulation of caspases in their study. Aurilide represents the first natural product that inhibits the function of prohibitin in the regulation of OPA1 and mitochondria-induced apoptosis.

In summary, further biological testing, including biochemical studies, allowed preliminary investigation of the lagunamides in terms of their cytotoxic activities and effects on programmed cell death. Lagunamide A exhibited selectivity in activity when tested against a panel of cell lines, including P388, A549, PC3, HCT8, and SK-OV3, with $\mathrm{IC}_{50}$ values ranging from $1.6 \mathrm{nM}$ to $6.4 \mathrm{nM}$. Although compound $\mathbf{2}$ was tested in only two cell lines, it showed lower potency when compared to $\mathbf{1}$, indicating the importance of the saturated form in the polyketide moiety of $\mathbf{1}$. Biochemical studies using HCT8 and MCF7 cancer cells suggested that the cytotoxic effect of the lagunamides might act via induction of mitochondrial mediated apoptosis. Data presented in this study underscores the importance of the aurilide class of molecules as potential anti-cancer therapeutic agents and also encourages more in-depth investigation into other therapeutic uses of this interesting class of marine derived compounds.

\section{Experimental Section}

\subsection{Extraction and Isolation of Lagunamides $A$ and $B$}

The filamentous benthic marine cyanobacterium, Lyngbya majuscula, was collected from the western lagoon of Pulau Hantu Besar, Singapore on 25 June, 2007. Voucher specimen of this strain is maintained at the National Institute of Education under the code TLT/PHB/002. The isolation and purification of the lagunamides follow that of Tripathi et al. [9]. Briefly, samples of the marine cyanobacterium, Lyngbya majuscula, stored previously in $70 \%$ aqueous EtOH, were extracted exhaustively with $1: 1 \mathrm{CHCl}_{3}: \mathrm{MeOH}$ to yield about $1.0 \mathrm{~g}$ of organic extract. The extract was subjected to normal phase Si gel vacuum flash chromatography and the bioactive fractions were identified using the brine shrimp toxicity assay. Upon clean up with RP-18 SEP-PAK, the active sub-fraction was subjected to further purification using RP-18 (Phenomenex Sphereclone $5 \mu \mathrm{m}$ ODS, $250 \times 10.00 \mathrm{~mm}$ ) Preparative HPLC, eluted with 78:22 $\mathrm{MeOH}: \mathrm{H}_{2} \mathrm{O}$, to provide pure lagunamides A (1) and B (2).

\subsection{Cancer Cell Lines and Media}

HCT8, MCF7, A549, PC3, SK-OV3, P388, and BJ cells were obtained from the American Type Culture Collection (ATCC). BJ shp53 cells were made in-house [31]. Cells and cell lines were 
maintained in either Dulbecco's modified Eagle's medium (DMEM, Gibco, Carlsbad, CA, USA) or RPMI-1640 media, supplemented with 10\% fetal bovine serum (FBS, Hyclone, Waltham, MA, USA), 5\% L-glutamine (PAA, Pasching, Austria) and 1\% penicillin/streptomycin (PAA, Pasching, Austria), incubated at $37{ }^{\circ} \mathrm{C}$ and $5 \% \mathrm{CO}_{2}$.

\subsection{MTT Cytotoxicity Assay}

The cytotoxicity on cells was determined by MTT staining assay, according to an established reported procedure [12,14]. All compounds were dissolved in $100 \%$ DMSO at $1 \mathrm{mM}$ stock concentration. In the MTT assay, the compound stock solutions were diluted in cell culture medium containing 10\% DMSO and pipetted into cell culture wells. The final DMSO concentration was $1.25 \%$ in each well. A standard plating format was used on each microtiter plate. Six serial dilutions were made for each compound in order to generate a dose response curve. Each plate contained a blank well (cell-free medium-only well), solvent control wells which contained cells and $1.25 \%$ DMSO, drug control wells which contained drug and medium only and growth control wells which contained only cells in medium. There were three replicate wells for each compound dilution.

Adherent cells were seeded at 20,000 cells per well and cultured for $24 \mathrm{~h}$ before exposure to compounds for $24 \mathrm{~h}$. After incubation, cell viability was determined by the MTT dye reduction method [12] and measured at $570 \mathrm{~nm}$ using a microtiter plate reader (Biorad, 168-1000EDU). The inhibitory concentration, $\mathrm{IC}_{50}$, was defined as the effective concentration of compounds that caused $50 \%$ reduction of cell growth relative to solvent controls. Each $\mathrm{IC}_{50}$ value was calculated based on the dose response curve by non-linear regression and sigmoidal analysis function in the GraphPad Prism data analysis software (version 5, GraphPad software: La Jolla, CA, USA, 2011).

\subsection{Western Blot Analysis}

Approximately 200,000 HCT8 and MCF7 cells were incubated with $100 \mathrm{nM}$ lagunamides A (1), $\mathrm{B}$ (2), and taxol for $10 \mathrm{~h}$. The cell pellets were collected by centrifugation at $200 \mathrm{~g}$ for $5 \mathrm{~min}$ at $4{ }^{\circ} \mathrm{C}$, washed three times with PBS and lysed by resuspension in lysis solution ( $7 \mathrm{M}$ urea, $2 \mathrm{M}$ thiourea, 4\% CHAPS, $30 \mathrm{mM}$ Tris, $\mathrm{pH} 9.0$ ) and brief sonication on ice for $2 \mathrm{~min}$ at 30\% amplitude, $30 \mathrm{~s}$ interval. The cell lysates were centrifuged at $12,000 \mathrm{rpm}$ for $5 \mathrm{~min}$. The protein-containing supernatants from the control untreated cells and treated cell lysates were quantified using Bradford protein assay reagent (Biorad, 500-0201). Equal amounts of proteins (20 mg) were boiled at $95{ }^{\circ} \mathrm{C}$ with $10 \mu \mathrm{L}$ of $2 \times$ laemmli sample buffer (Biorad, 161-0737) for 5 min and loaded into each lane of a SDS-PAGE gel. Cell lysates were separated by pre-cast 4-15\% SDS-PAGE under reducing conditions and transferred to nitrocellulose membranes. Membranes were blocked with 5\% non-fat milk diluted in TBS-T for $1 \mathrm{~h}$ at room temperature. The membranes were then probed with rabbit anti-caspase-3 (1:1000), rabbit anti-caspase-9 (1:1000) or rabbit anti-Bcl-xl (1:1000) antibodies (Cell Signaling Technology) at $4{ }^{\circ} \mathrm{C}$ overnight. Mouse anti-Beta-actin $(1: 20,000)$ (Santa Cruz) was used as a loading control. Incubation with anti-Beta-actin antibodies was for $1 \mathrm{~h}$ at room temperature. After washing with TBS-T, the membranes were incubated with goat anti-rabbit HRP-conjugated antibody $(1: 40,000)$ (Santa Cruz) or goat anti-mouse HRP conjugated antibody $(1: 40,000)$ (Santa Cruz). Specific band signals were detected by chemiluminescence on film. 


\section{Conclusions}

Biological studies of the lagunamides, in particular compound $\mathbf{1}$, showed selective cytotoxicity against a panel of cancer cell lines, with HCT8 being the most sensitive cell line with an $\mathrm{IC}_{50}$ of $1.8 \mathrm{nM}$. Preliminary data from a series of biochemical experiments using HCT8 and MCF7 cell lines suggested that the cytotoxic effects of the lagunamides operate via the intrinsic apoptotic pathway.

\section{Acknowledgments}

The authors would like to acknowledge NIE AcRF (RI 11/10 TLT) and NUS AcRF (Tier 1/R-279-000-321-133 to D.T.L.) for their funding support.

\section{References}

1. Tan, L.T. Bioactive natural products from marine cyanobacteria for drug discovery. Phytochemistry 2007, 68, 954-979.

2. Cane, D.E.; Walsh, C.T.; Khosla, C. Harnessing the biosynthetic code: Combinations, permutations, and mutations. Science 1998, 282, 63-68.

3. Tan, L.T. Filamentous tropical marine cyanobacteria: A rich source of natural products for anticancer drug discovery. J. Appl. Phycol. 2010, 22, 659-676.

4. Minich, S.S. Brentuximab vedotin: A new age in the treatment of Hodgkin lymphoma and anaplastic large cell lymphoma. Ann. Pharmacother. 2012, 46, 377-383.

5. Gerwick, W.H.; Moore, B.S. Lessons from the past and charting the future of marine natural products drug discovery and chemical biology. Chem. Biol. 2012, 19, 85-98.

6. Tan, L.T.; Chang, Y.Y.; Tripathi, A. Besarhanamides A and B from the marine cyanobacterium Lyngbya majuscula. Phytochemistry 2008, 69, 2067-2069.

7. Tripathi, A.; Puddick, J.; Prinsep, M.R.; Lee, P.P.; Tan, L.T. Hantupeptin A, a cytotoxic cyclic depsipeptide from a Singapore collection of Lyngbya majuscula. J. Nat. Prod. 2009, 72, 29-32.

8. Tripathi, A.; Puddick, J.; Prinsep, M.R.; Lee, P.P.; Tan, L.T. Hantupeptins B and C, cytotoxic cyclodepsipeptides from the marine cyanobacterium Lyngbya majuscula. Phytochemistry 2010, 71, 307-311.

9. Tripathi, A.; Puddick, J.; Prinsep, M.R.; Rottmann, M.; Tan, L.T. Lagunamides A and B: cytotoxic and antimalarial cyclodepsipeptides from the marine cyanobacterium Lyngbya majuscula. J. Nat. Prod. 2010, 73, 1810-1814.

10. Taraphdar, A.K.; Roy, M.; Bhattacharya, R.K. Natural products as inducers of apoptosis: Implication for cancer therapy and prevention. Curr. Sci. 2001, 80, 1387-1396.

11. Wasilewski, M.; Scorrano, L. The changing shape of mitochondrial apoptosis. Trends Endocrinol. Metab. 2009, 20, 287-294.

12. Hanahan, D.; Weinberg, R.A. The hallmarks of cancer. Cell 2000, 100, 57-70.

13. Nakao, Y.; Yoshida, W.Y.; Takada, Y.; Kimura, J.; Yang, L.; Mooberry, S.L.; Scheuer, P.J. Kulokekahilide-2, a cytotoxic depsipeptide from a cephalaspidean mollusk Philinopsis speciosa. J. Nat. Prod. 2004, 67, 1332-1340. 
14. Han, B.; Gross, H.; Goeger, D.E.; Mooberry, S.L.; Gerwick, W.H. Aurilides B and C, cancer cell toxins from a Papua New Guinea collection of the marine cyanobacterium Lyngbya majuscula. J. Nat. Prod. 2006, 69, 572-575.

15. Takada, Y.; Umehara, M.; Nakao, Y.; Kimura, J. Revised absolute stereochemistry of natural kulokekahilide-2. Tetrahedron Lett. 2008, 49, 1163-1165.

16. Suenaga, K.; Mutou, T.; Shibata, T.; Itoh, T.; Kigoshi, H.; Yamada, K. Isolation and stereostructure of aurilide, a novel cyclodepsipeptide from the Japanese sea hare Dolabella auricularia. Tetrahedron Lett. 1996, 37, 6771-6774.

17. Suenaga, K.; Mutou, T.; Shibata, T.; Itoh, T.; Fujita, T.; Takada, N.; Hayamizu, K.; Takagi, M.; Irifune, T.; Kigoshi, H.; et al. Aurilide, a cytotoxic depsipeptide from the sea hare Dolabella auricularia: Isolation, structure determination, synthesis, and biological activity. Tetrahedron 2004, 60, 8509-8527.

18. Takashi, T.; Nagamiya, H.; Doi, T.; Griffiths, P.G.; Bray, A.M. Solid phase library synthesis of cyclic depsipeptides: aurilide and aurilide analogues. J. Comb. Chem. 2003, 5, 414-428.

19. Suenaga, K.; Kajiwara, S.; Kuribayashi, S.; Handa, T.; Kigoshi, H. Synthesis and cytotoxicity of aurilide analogs. Bioorg. Med. Chem. Lett. 2008, 18, 3902-3905.

20. Takada, Y.; Mori, E.; Umehara, M.; Nakao, Y.; Kimura, J. Reinvestigation of the stereochemistry of kulokekahilide-2. Tetrahedron Lett. 2007, 48, 7653-7656.

21. Takada, Y.; Umehara, M.; Katsumata, R.; Nakao, Y.; Kimura, J. The total synthesis and structure-activity relationships of a highly cytotoxic depsipeptide kulokekahilide-2 and its analogs. Tetrahedron 2012, 68, 659-669.

22. Finlay, C.A.; Hinds, P.W.; Levine, A.J. The p53 proto-oncogene can act as a suppressor of transformation. Cell 1989, 57, 1083-1093.

23. Lowe, S.W.; Cepero, E.; Evan, G. Intrinsic tumour suppression. Nature 2004, 432, 307-315.

24. Fulda, S.; Debatin, K.-M. Extrinsic versus intrinsic apoptosis pathways in anticancer chemotherapy. Oncogene 2006, 25, 4798-4811.

25. Spierings, D.; McStay, G.; Saleh, M.; Bender, C.; Chipuk, J.; Maurer, U.; Green, D.R. Connected to death: The (unexpurgated) mitochondrial pathway of apoptosis. Science 2005, 310, 66-67.

26. Chen, Q.Y.; Liu, Y.; Luesch, H. Systematic chemical mutagenesis identifies a potent novel apratoxin A/E hybrid with improved in vivo antitumor activity. ACS Med. Chem. Lett. 2011, 2, 861-865.

27. O’Donnell, D.R.; Milligan, L.; Stark, J.M. Induction of CD95 (Fas) and apoptosis in respiratory epithelial cell cultures following respiratory syncytial virus infection. Virology 1999, 257, 198-207.

28. Wrasidlo, W.; Mielgo, A.; Torres, V.A.; Barbero, S.; Stoletov, K.; Suyama, T.L.; Klemke, R.L.; Gerwick, W.H.; Carson, D.A.; Stupack, D.G. The marine lipopeptide somocystinamide A triggers apoptosis via caspase 8. Proc. Nat. Acad. Sci. 2007, 105, 2313-2318.

29. Sato, S.; Murata, A.; Orihara, T.; Shirakawa, T.; Suenaga, K.; Kigoshi, H.; Uesugi, M. Marine natural product aurilide activates the OPA1-mediated apoptosis by binding to prohibitin. Chem. Biol. 2011, 18, 131-138.

30. Mishra, S.; Murphy, L.C.; Nyomba, B.L.; Murphy, L.J. Prohibitin: A potential target for new therapeutics. Trends Mol. Med. 2005, 11, 192-197. 
31. Ng, K.W.; Khoo, S.P.; Heng, B.C.; Setyawati, M.I.; Tan, E.C.; Zhao, X.; Xiong, S.; Fang, W.; Leong, D.T.; Loo, J.S. The role of the tumor suppressor p53 pathway in the cellular DNA damage response to zinc oxide nanoparticles. Biomaterials 2011, 32, 8218-8225.

Samples Availability: Available from the authors.

(C) 2012 by the authors; licensee MDPI, Basel, Switzerland. This article is an open access article distributed under the terms and conditions of the Creative Commons Attribution license (http://creativecommons.org/licenses/by/3.0/). 